\title{
The occurrence and damage of unipolar arcing on fuzzy tungsten
}

\author{
D.U.B. Aussems ${ }^{\mathrm{a} *}$, D. Nishijima ${ }^{\mathrm{b}}$, C. Brandt ${ }^{\mathrm{b}}$, H.J. van der Meiden ${ }^{\mathrm{a}}$, M. Vilémovác, \\ J. Matějíček ${ }^{\mathrm{c}}$, G. De Temmerman ${ }^{\mathrm{a}}$, R.P. Doerner ${ }^{\mathrm{b}}$, N. J. Lopes Cardozo ${ }^{\mathrm{d}}$ \\ ${ }^{a}$ FOM Institute DIFFER-Dutch Institute for Fundamental Energy Research, Nieuwegein, the \\ Netherlands, Nieuwegein, NL-3430 BE, the Netherlands \\ ${ }^{b}$ Center for Energy Research, University of California at San Diego, 9500 Gilman Drive, La Jolla, CA \\ 92093-0417, USA \\ ${ }^{\mathrm{c}}$ Institute of Plasma Physics, Association EURATOM-IPP, 18200 Prague 8, Czech Republic \\ ${ }^{d}$ Science and Technology of Nuclear Fusion, Eindhoven University of Technology, Den Dolech 2, \\ Eindhoven, the Netherlands
}

\begin{abstract}
This research investigated whether unipolar arcing in the divertor of fusion reactors is a potential cause for enhanced wear of the divertor. It was found that $1 \mu \mathrm{m}$ of nano-fuzz growth is sufficient to initiate arcing, mainly depending on the sheath potential drop and electron density. The average mass loss rate induced by the arc was determined from mass loss measurements and found to be consistent with the value estimated from the arc current. The average arc track erosion depth was estimated by using the measured mass loss and damaged surface area and was found to be one tenth of the fuzzy layer thickness. Due to melting of the fuzzy structures the actual depth is larger and some arc tracks occasionally appeared to even reach the bulk beyond the fuzzy layer. The conclusion of this study is therefore that arcing in the divertor of future tokamaks (e.g. ITER) potentially is an important cause for surface damage and plasma pollution.
\end{abstract}

PACS: 79.20.Rf, 52.40.Hf, 52.55.Fa, 52.25.Gj 
PSI-21 keywords: unipolar arcing, fuzzy tungsten

*Corresponding author address: FOM Institute DIFFER, P.O. Box 1207, NL-3430 BE, Nieuwegein, the Netherlands.

*Corresponding author E-mail: $\underline{\text { d.aussems @ differ.nl }}$

Presenting author: Damien Aussems

Presenting author e-mail: d.aussems@differ.nl 


\section{Introduction}

Tungsten will be used as divertor material in ITER. Recent experiments show that helium plasma exposure on tungsten can lead to nano-scale structure formation referred to as 'fuzz', even when the incident ion energy is less than the threshold energy for physical sputtering [1]. In fact, the growth of fuzz has recently been observed in the divertor of the Alcator C-Mod tokamak [2]. Fuzz may significantly increase the occurrence of unipolar arcing due to transient heat loads (e.g. ELMs) on the divertor [3].

The existence of the unipolar arc has been confirmed phenomenologically by erosion craters found in numerous fusion devices [4][5][6][7]. Arcing on fuzzy tungsten has recently been investigated in special model experiments using amongst others a plasma gun [8] and a laser pulse [9].

Unipolar arc behavior has not been investigated sufficiently in an divertor relevant environment in which amongst others the sample temperature, sheath potential and electron density can be varied. This research aims to investigate the conditions for which arcing on fuzz tungsten may occur, and how the damage can be related to those conditions. Arcing is investigated in a fusion relevant environment using the PISCES-A and MAGNUM-PSI linear plasma devices, which enable us to easily access the plasma and tungsten sample.

\section{Experimental setup}

Most of the work done in this research has been performed on PISCES-A, while some supporting experiments were done on MAGNUM-PSI.

\subsection{PISCES-A}

The linear plasma device PISCES-A is described in detail in Ref. [10]. The typical plasma is generated with a density of $>10^{18} \mathrm{~m}^{-3}$ and electron temperature of $5-7 \mathrm{eV}$ (measured by a plunging Langmuir probe [11]). The plasma flows along the magnetic field to the target 
where the ions are accelerated by a sheath potential on an electrically biased target, producing an ion flux on the order of $10^{22} \mathrm{~m}^{-2} \mathrm{~s}^{-1}$ with an ion energy that can be controlled between $50-250 \mathrm{eV}$. The target temperature was set to $\sim 1200 \mathrm{~K}$ by controlling the cooling air flow. The experimental setup is depicted in Figure 1. In each campaign, a fuzz layer $(\sim 1 \mu \mathrm{m})$ was created by exposing the target to a He-plasma for $1 \mathrm{hr}$ at a fixed sample temperature between $1170-1220 \mathrm{~K}$, and bias voltage of $-100 \mathrm{~V}$ and $-60 \mathrm{~V}$ in campaign 1 and 2 , respectively. Quickly before the onset of the arcing, the relevant experimental parameters are varied. To initiate arcing, a $1064 \mathrm{~nm} \mathrm{Nd-YAG} \mathrm{laser} \mathrm{pulse} \mathrm{is} \mathrm{focused} \mathrm{on} \mathrm{the} \mathrm{target} \mathrm{(max.} \mathrm{laser} \mathrm{power}$ density $1.1 \times 10^{14} \mathrm{~W} \mathrm{~m}^{-2}, 6 \mathrm{~ns}$ pulse, spot size $\left.1 \mathrm{~mm}\right)$.

A fast camera (Phantom I) is installed at the right side view port to observe the arcs on the target. To calculate the transient current during arcing, a Pearson current monitor is installed between the target and the ground. After each experiment the tungsten sample is removed from PISCES-A, and photographed by a handheld camera. An image analysis program is used to determine the damaged surface area. The mass of the sample is measured in threefold (for statistics) before and after each experiment using a high precision scale (10 $\mu \mathrm{g}$ min. scale).

\subsection{MAGNUM-PSI}

The experimental setup of Magnum-PSI plasma device is described in [12]. In MAGNUMPSI a plasma is generated with a typical high plasma density $\left(>10^{19} \mathrm{~m}^{-3}\right)$ and electron temperature of $1-2 \mathrm{eV}$ (measured by a Thomson Scattering system [13]). The plasma is radially confined by the magnetic field of $0.8 \mathrm{~T}$ and flows to a water-cooled, electrically biased target, achieving a particle flux of $1.5-3 \times 10^{23} \mathrm{~m}^{-2} \mathrm{~s}^{-1}$, ion energy of 25-65 eV, and surface temperature of $500-720 \mathrm{~K}$. For the arc experiments, fuzzy W samples were first made in PISCES-A (the plasma diameter is larger in PISCES and allows for a more uniform fuzz surface), and then installed in MAGNUM-PSI. 
Initiation of arcing was achieved in a similar fashion as in PISCES-A, this time using two different lasers systems (for pulse width comparison). The first is a $1064 \mathrm{~nm}$ fibre-coupled Nd:YAG laser (pulse width $1 \mathrm{~ms}$, power density $1.0 \times 10^{8} \mathrm{Wm}^{-2}$, spot size $1 \mathrm{~mm}$ ). The other laser system is a $1064 \mathrm{~nm} \mathrm{Nd:YAG} \mathrm{laser} \mathrm{with} \mathrm{a} \mathrm{conventional} \mathrm{beam} \mathrm{line} \mathrm{(pulse} \mathrm{width} 8$ ns, power density $4.8 \times 10^{12} \mathrm{Wm}^{-2}$, spot size $\left.1 \mathrm{~mm}\right)$.

The surface temperature of the target during the experiment is determined by an IR-camera (FLIR SC7500MB). The fuzz emissivity was measured ex-situ in the wavelength range 3.97-4.01 $\mu \mathrm{m}$ and equals approximately 2.2 times the emissivity of pristine tungsten.

\section{Arc observation}

The fast camera emission images (side view) of a typical arc in PISCES-A are shown in Fig. 2 for different delay times (exposure time is $7.8 \mu \mathrm{s}$ ). The unipolar arc is ignited by a laser pulse which induces a dense tungsten plasma. After $\sim 40 \mu$ s this plasma has been significantly expanded and the unipolar arc plasma becomes dominant. The arc splits into two emission spots, which start to randomly move around the surface. At $2.56 \mathrm{~ms}$ droplets are emitted from the surface and finally the arc spot extinguishes after $2.67 \mathrm{~ms}$.

The arcs have a duration of 2-6 ms and sustain a current of 20-70 A. The maximum emission intensity is caused by the laser-induced plasma, and occurs during the first $100 \mu \mathrm{s}$, and is at least a factor 10 higher than the average intensity during the arc. A typical arc propagation speed was found of $\sim 50 \mathrm{~ms}^{-1}$. The light emission area (side view as in Fig. 2) was $50 \pm 30 \mathrm{~mm}^{2}$ on average.

For some shots we were able to observe droplets. The droplets mainly originated from the edges of the target and moved with a speed $\sim 10 \mathrm{~ms}^{-1}$ from the arc emission center, one order of magnitude lower than the literature value $100 \mathrm{~ms}^{-1}$ (Ref. [14]). 


\section{Arc occurrence}

Several parameters influencing the arc ignition threshold have been investigated, and have found to be mutually related. For example in some cases the bias voltage can be smaller, if the electron density is higher. For this reason the threshold value has to be viewed considering the other parameters.

Bias voltage / sample temperature. Table 1 shows the occurrence of arcing as function of the bias voltage and sample temperature in PISCES-A $\left(P_{\text {laser }}=7.5 \times 10^{13} \mathrm{Wm}^{-2}, n_{e}=2.5 \times\right.$ $\left.10^{18} \mathrm{~m}^{-3}\right)$

First of all, the occurrence of arcing highly depends on the bias voltage. During our experiments in PISCES-A arcing never occurred at $\left|V_{\text {bias }}\right|<-80 \mathrm{~V}$. In the temperature range 970 to $1220 \mathrm{~K}$ the occurrence of arcing depends on the bias voltage only. The lower $\left|V_{\text {bias }}\right|$ threshold for arcing at $T_{S}=1270 \mathrm{~K}$ can be either explained by the shot-to-shot variation or a more intense primary erosion plasma due to a higher base temperature. The lower threshold at $T_{S}=870 \mathrm{~K}$ was unexpected and needs to be confirmed.

Pressurelelectron density. In an experiment in PISCES-A $\left(P_{\text {laser }}=7.5 \times 10^{13} \mathrm{Wm}^{-2}\right.$, $V_{\text {bias }}=-100 \mathrm{~V}, T_{s}=1120 \mathrm{~K}$ ) the neutral gas pressure was decreased from 1.0 to $0.3 \mathrm{~Pa}$, corresponding to an electron density drop from $2.5 \times 10^{18} \mathrm{~m}^{-3}$ to $9.0 \times 10^{17} \mathrm{~m}^{-3}$, and arcing no longer occurred.

In an experiment in MAGNUM-PSI the electron density was $3.4 \times 10^{19} \mathrm{~m}^{-3}$ and arcing already occurred at a bias voltage of $-65 \mathrm{~V}\left(T_{s}=710 \mathrm{~K}\right)$, using a similar laser with pulse width $8 \mathrm{~nm}$ and power density $9.2 \times 10^{12} \mathrm{Wm}^{-2}$ (one order of magnitude lower than PISCES-A). 
The $35 \mathrm{~V}$ smaller bias voltage at which arcing occurs in MAGNUM-PSI can be related to the fact that the electron density is one order of magnitude higher. A higher density decreases the (Child) sheath thickness $S_{\text {Child }}=\sqrt{2} \lambda_{D e} / 3 \times\left(2 e \mid \Delta V /\left(k T_{e}\right)\right)^{3 / 4}$, in which $\lambda_{D e}=$ $\sqrt{\varepsilon_{0} k T_{e} /\left(n_{e} e^{2}\right)}$ [14]. To reach the same electron emission, and thus local electric field (roughly approximated as $E_{\text {surf }}=4 V_{c} /\left(3 s_{\text {Child }}\right)$, in which $\mathrm{V}_{\mathrm{c}}$ is the cathode fall), the bias voltage can be less negative. This can also explain the electron density dependence which we observed in our experiments.

Remarkably, this electron density dependence was not observed under the conditions of previous work on NAGDIS-II [9]. A possible explanation is that the fuzz thickness is larger than in PISCES-A, because the surface temperature during exposure was significantly higher ( $1630 \mathrm{~K}$ versus $\sim 1200 \mathrm{~K}$ ). This can ease the triggering of arcing and thus, under the experimental conditions used in Ref. [9], the influence of the electron density may be negligible.

Laser pulse width. Up till now, nanosecond laser pulses were used to ignite arcing, although a single ELM event has a millisecond duration. To investigate whether arcing still occurs at longer pulse duration, two laser systems with different pulse widths of $1 \mathrm{~ms}$ and $8 \mathrm{~ns}$ were used in MAGNUM-PSI. Under similar experimental conditions $\left(n_{e}=4.2\right.$ vs $3.4 \times$ $10^{19} \mathrm{~m}^{-3}, T_{s}=710 \mathrm{vs} 510 \mathrm{~K}, V_{\text {bias }}=-65 \mathrm{vs}-55 \mathrm{~V}$, respectively), it was found that arcing is ignited if the heat load impact factor $(\mathrm{HF})$ was of the same order of magnitude, but a factor 2.6 off (2.3 vs $8.3 \times 10^{8} \mathrm{Jm}^{-2} \mathrm{~s}^{-1 / 2}$, respectively).

The smaller bias voltage in the case of the ns laser can be explained by both the higher HF and power density $\left(1.0 \times 10^{10}\right.$ vs $\left.9.2 \times 10^{12} \mathrm{Wm}^{-2}\right)$. This last quantity activates different processes on the target which influence arc occurrence; for power densities exceeding 
$10^{12} \mathrm{Wm}^{-2}$ evaporation is replaced by plasma generation as a dominant heat coupling mechanism [15].

\section{Target damage}

\subsection{Results}

The target damage due to arcing was predominantly investigated in PISCES-A. In addition, surface analysis was performed on samples from MAGNUM-PSI.

Surface analysis. Fig. 3 a) shows the damage due to the laser shot and arcing in MAGNUMPSI. The black surface is fuzz. The high porosity of the fuzz causes reflectivity to be almost zero (for visible wavelength range) [16]. The bright area is the laser strike spot where the arcing starts. From this spot, an arc track to the left direction can be observed, until it stops at the edge. Fig. 3 b)-d) show that the fuzz layer is damaged due to arcing. In PISCES-A, when the fuzz is carefully polished with a Kimwipe ( ) (until the bulk material is visible) some arc tracks were still visible.

Mass loss. An average target mass difference before and after the experiment was measured, and was found to be $253 \pm 48 \mu \mathrm{g}$ with arcing, and $189 \pm 46 \mu \mathrm{g}$ without arcing. This means that the mass loss attributed to arcing is $64 \pm 67 \mu \mathrm{g}$. Because of the high uncertainty of the measurements, however, it is hard to distinguish between mass loss due to ion sputtering and due to arcing.

Current. The mass loss was also determined using an estimation of the mass loss per charge $\gamma_{i}[\mu \mathrm{g} / \mathrm{C}]$, and measuring the charge $\left(C=\int_{0}^{t} I\left(t^{*}\right) d t^{*}\right)$. The value for the mass loss per charge found in recent experiments is $\gamma_{i}=1 \mathrm{mg} / \mathrm{C}$ [9] (used in for this work), which is 
(unexpectedly) two orders of magnitude higher than theoretically estimated [17]. An example of the measured current is given in Fig. 4. Within the first $0.5 \mathrm{~ms}$ the current is the highest and slowly decreases to an approximately steady-state value. At the end of the arc, the current drops down to zero.

The mass loss calculated from the measured current is $15-95 \mu \mathrm{g}$, which is close to the average mass loss of $\sim 60 \mu \mathrm{g}$ found by the direct measurements. The mass loss calculated from the current only includes the ion mass loss. Nevertheless, the contributions of neutrals and droplets to the mass loss are expected to be low; (1\%, and $3.5 \%$, respectively [14]).

Damaged surface area. The damaged surface area due to arcing is $1-4 \mathrm{~cm}^{2}$. In general, the shots show that the damaged surface area is proportional to the mass loss due to arcing calculated from the current measurements.

\subsection{Discussion}

The rate of damaged surface area can be calculated by dividing the damaged surface area by the arc duration. The average rate of all shots is $0.9 \pm 0.3 \times 10^{-2} \mathrm{~m}^{2} \mathrm{~s}^{-1}$, which is the same order of magnitude as the value estimated in Ref. [9].

The effective depth in fuzz due to arcing might be calculated from the damaged surface area, and the mass loss determined by the integrated arc current. Assuming a porosity of $90 \%$ [18] the tungsten fuzz density is $1925 \mathrm{kgm}^{-3}$, and the average arc track erosion depth in fuzz obtained is $100 \pm 30 \mathrm{~nm}$, which is about one tenth of the fuzz layer, and a factor two higher than found by Ref. [9]. The actual arc damage depth may be significantly deeper due to melting and thus conglomeration of fuzzy structures. Some arc tracks were found to penetrate even into the bulk material. 
The associated mass loss rate (mass per arc duration) is $1-3 \times 10^{-2} \mathrm{gs}^{-1}$, which is similar to the value from Ref. [9]. In comparison, the mass loss rate found in our experiments is similar to the value currently anticipated for the divertor in ITER of $1.75 \times 10^{-2} \mathrm{gs}^{-1}$ during steadystate operation [19]. It should be noted that the effects of non-normal magnetic field incidence was not investigated. Moreover, the damage may be more severe for the high sheath potentials (max. $600 \mathrm{~V})$ expected during an ELM [20].

\section{Conclusion}

The damage of unipolar arcing on fuzzy tungsten was investigated in an environment similar to the divertor plate of a fusion reactor. It was found that if tungsten fuzz grows, arcing can occur mainly depending on the sheath potential and electron density. Locally, arcing can potentially lead to significant damage and the release of tungsten dust and droplets into the plasma. The conclusion of this study therefore is that arcing potentially is an important cause for damage in future tokamaks such as ITER. Therefore it needs to be investigated more comprehensively and mitigation measures must be developed, such as fuzz prevention.

\section{Acknowledgements}

The authors wish to thank PISCES and DIFFER technical staff for their professional skill and dedicated support. This work was supported by the US DOE under the contract DE-FG0207ER54912, the EURATOM mobility funds, FOM Program 148, and grant no. 14-12837S of the Czech Science Foundation.

\section{Bibliography}

[1] S. Takamura, N. Ohno, D.Nishijima, et al., J. Plasma Fusion Res. 1 (2006) 051.

[2] G. Wright, M. B. D. Brunner, et al., Nucl. Fusion 52 (2012) 042003. 
[3] S. Kajita, S. Takamura, N. Ohno, Nucl. Fusion 49 (2009) 032002.

[4] S. Kajita, M. Fukumoto, M. Tokitani, et al., Nucl. fusion 53 (2013) 053013.

[5] D. Rudakov, C. Chrobak, R. Doerner, et al., J. Nucl. Mater. 438 (2013) S805.

[6] V. Rohde, N. Endstrasser, U. Toussaint, et al., J. Nucl. Mater. 415 (2011) S46.

[7] A. Herrmann, M. Balden, M. Laux, et al., J. Nucl. Mater. 390-391 (2009) 747.

[8] D. Nishijima, Y. Kikuchi, M. Nakatsuka, et al., Fusion Sci. Techn. 60 (4) (2011) 1447.

[9] S. Kajita, N. Ohno, N. Yoshida, et al., Plasma Phys. Contr. Nucl. Fusion 54 (2012) 035009.

[10] D. Aussems, D. Nishijima, C. Brandt, et. al., Appl. Phys. (in press).

[11] E. M. Hollmann, C. Brandt, B. Hudson, et al., Phys. Plasmas 20 (2013) 093303-9

[12] G. D. Temmerman, M. van den Berg, J. Scholten, et al., Fusion Eng. Des. 88 (2013) 483.

[13] H. J. van der Meiden, A. R. Lof, M. A. van den Berg, et al, Rev. Sci. Ins. 83 (2012) 123505

[14] A. Anders, Springer, Cathodic Arcs, Springer, New York, 2008.

[15] J. Singh, S. Thakur, Laser Induced Breakdown Spectroscopy, Oxford, Elsevier, 2007.

[16] D. Nishijima, R. Doerner, D. Iwamoto, et al., J. Nucl. Mater. 434 (2013) 230.

[17] S. A. Barengolts, G. A. Mesyats, M. M. Tsventoukh, in: 30th ICPIG, 2011.

[18] D. Nishijima, M. Baldwin, R. Doerner, et al., J. Nucl. Mater. 415 (2011) 1447.

[19] J. Roth, E. Tsitroneb, A. Loarted, et al., J. Nucl. Mater. 390-391 (2009) 1.

[20] A. Donné, A. Costley, R. Barnsley, et al., Nucl. Fusion 47 (2007) S337.

\section{Figure captions}

Figure 1: Illustration of the arcing experiment in PISCES-A. A tungsten target is exposed to helium plasma and a fuzz layer is grown. The laser hits the fuzzy tungsten target and arcing is initiated if the conditions are within the parameter space for arcing. 
Figure 2: Fast camera emission images of the arcing in PISCES-A for different delay times. The arc is initiated by a laser pulse on a fuzzy tungsten target. The images show arc splitting in multiple emission spots, arc propagation, droplet formation and finally the extinction of the arc.

Figure 3: a) Photograph of the damage due to laser shot and resulted arc tracks from an experiment in MAGNUM-PSI. b) and c) close-up SEM images of the arc tracks show that the original fuzz $\left(4^{*}\right)$ is melted due to $\operatorname{arcing}\left(5^{*}\right)$ but not removed. d) The arc tracks $\left(3^{*}\right)$ start in the region between the laser-damaged area $\left(1^{*}\right)$ and the original fuzz area (left side $2^{*}$ ).

Figure 4: Measured target current during a typical arc.

\section{Figures}


Figure 1

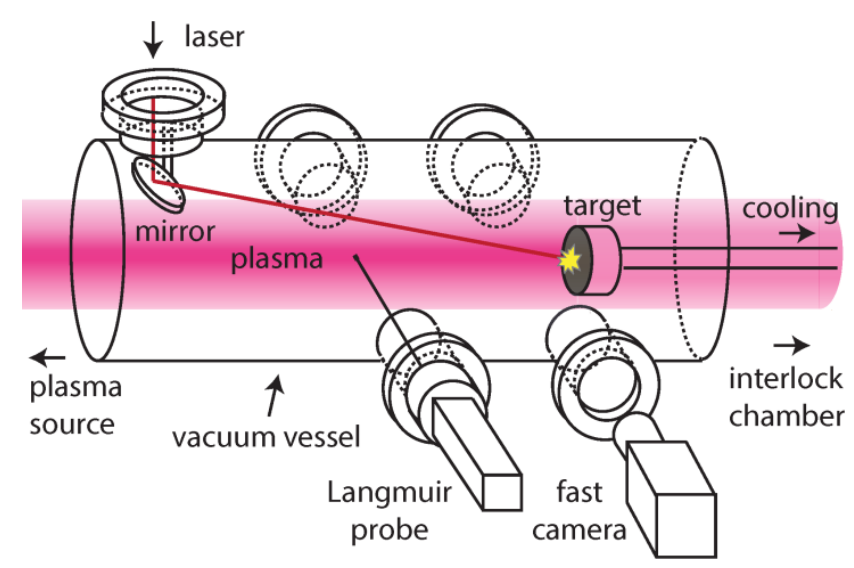


Figure 2

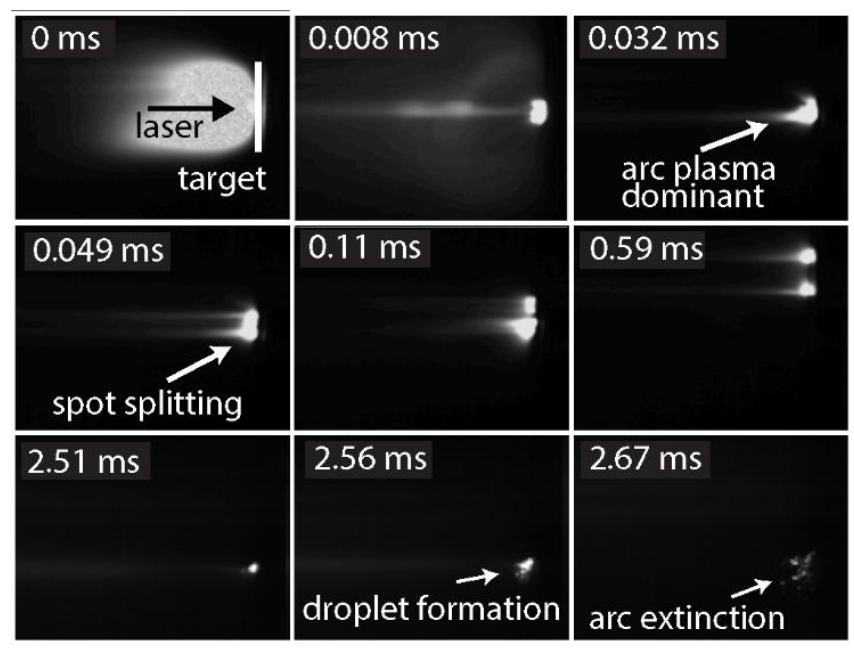


Figure 3

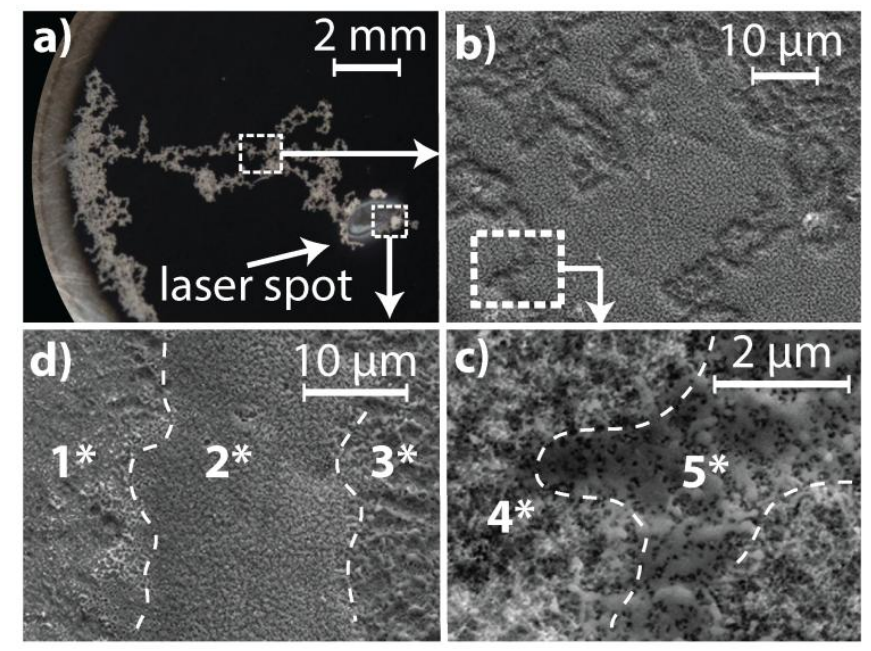


Figure 4

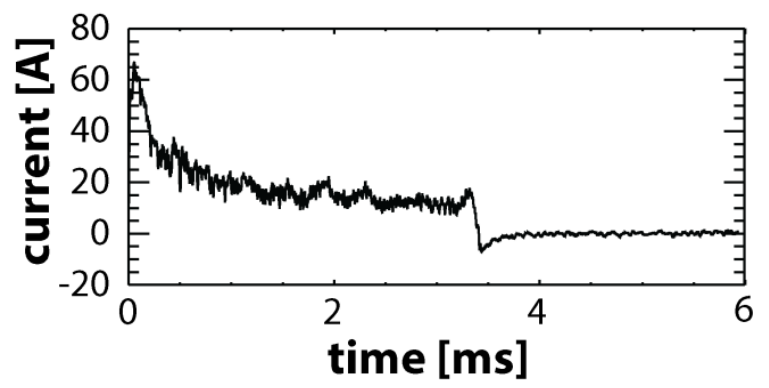




\section{Table caption}

Table 1: The occurrence of $\operatorname{arcing}\left(' \mathrm{Y}^{\prime}=\right.$ yes, and ' $\mathrm{N}$ ' = no) as function of the bias voltage, and sample temperature. Campaign 2 is indicated with $\left(^{*}\right)$.

\section{Tables}

Table 1

\begin{tabular}{cccccccc}
\hline$V_{\text {bias }}$ & \multicolumn{6}{c}{$T_{S}[\mathrm{~K}]$} \\
\cline { 2 - 8 } [V] & 870 & 970 & 1070 & 1120 & 1170 & 1220 & 1270 \\
\hline-160 & & & & & $\mathrm{Y}$ & & \\
-140 & & & & & $\mathrm{Y}$ & $\mathrm{Y}^{*}$ & \\
-120 & & & & & $\mathrm{Y}$ & $\mathrm{Y}^{*}$ & \\
-100 & $\mathrm{Y}$ & $\mathrm{Y}$ & $\mathrm{Y}$ & $\mathrm{Y}^{*}$ & $\mathrm{Y}^{*}$ & $\mathrm{Y}^{*}$ & $\mathrm{Y}$ \\
-80 & $\mathrm{Y}^{*}$ & $\mathrm{~N}$ & $\mathrm{~N}^{*}$ & & $\mathrm{~N}$ & $\mathrm{~N}^{*}$ & $\mathrm{Y}$ \\
-60 & $\mathrm{~N}^{*}$ & & & & & $\mathrm{~N}^{*}$ & $\mathrm{~N}$ \\
\hline
\end{tabular}

